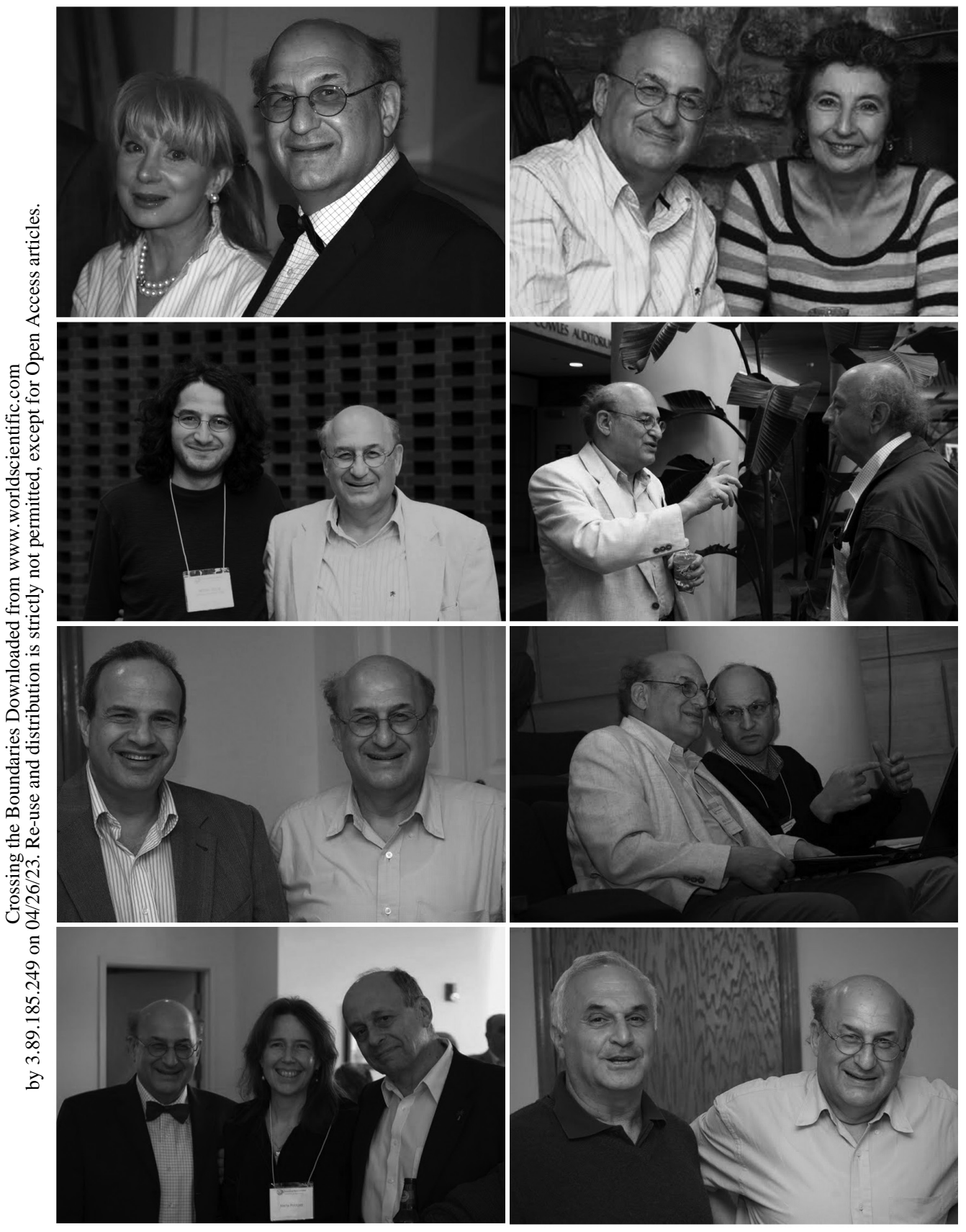




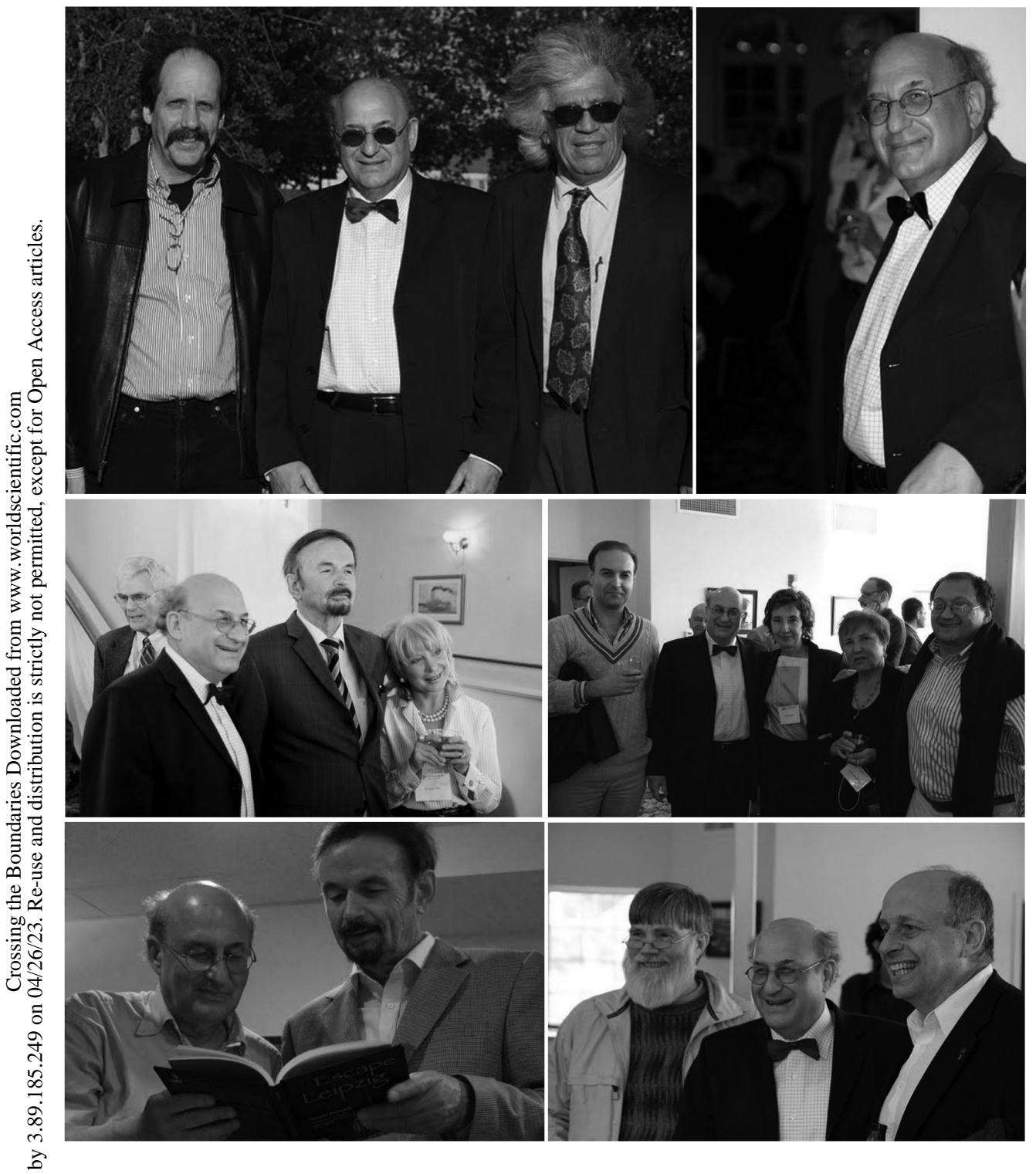




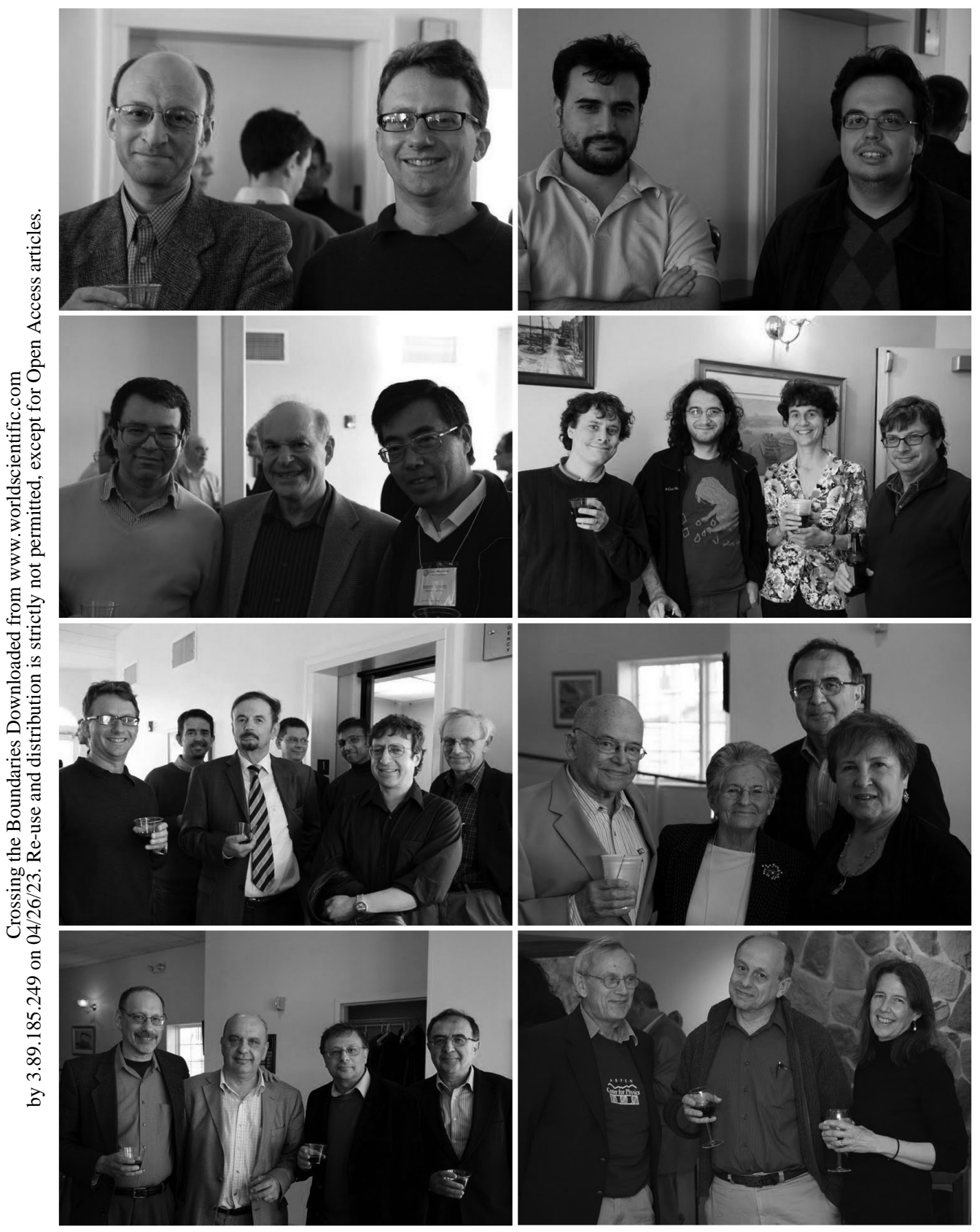




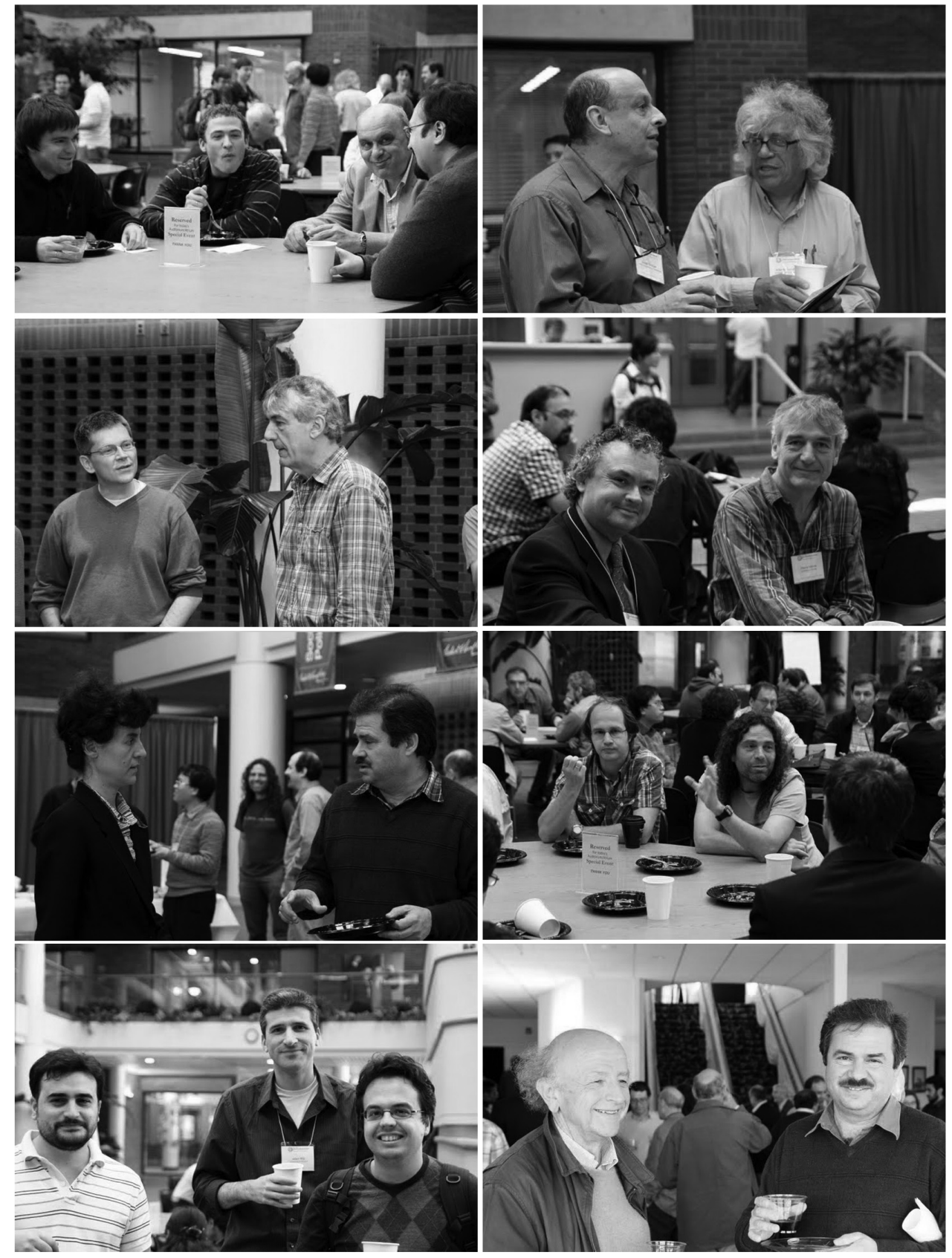




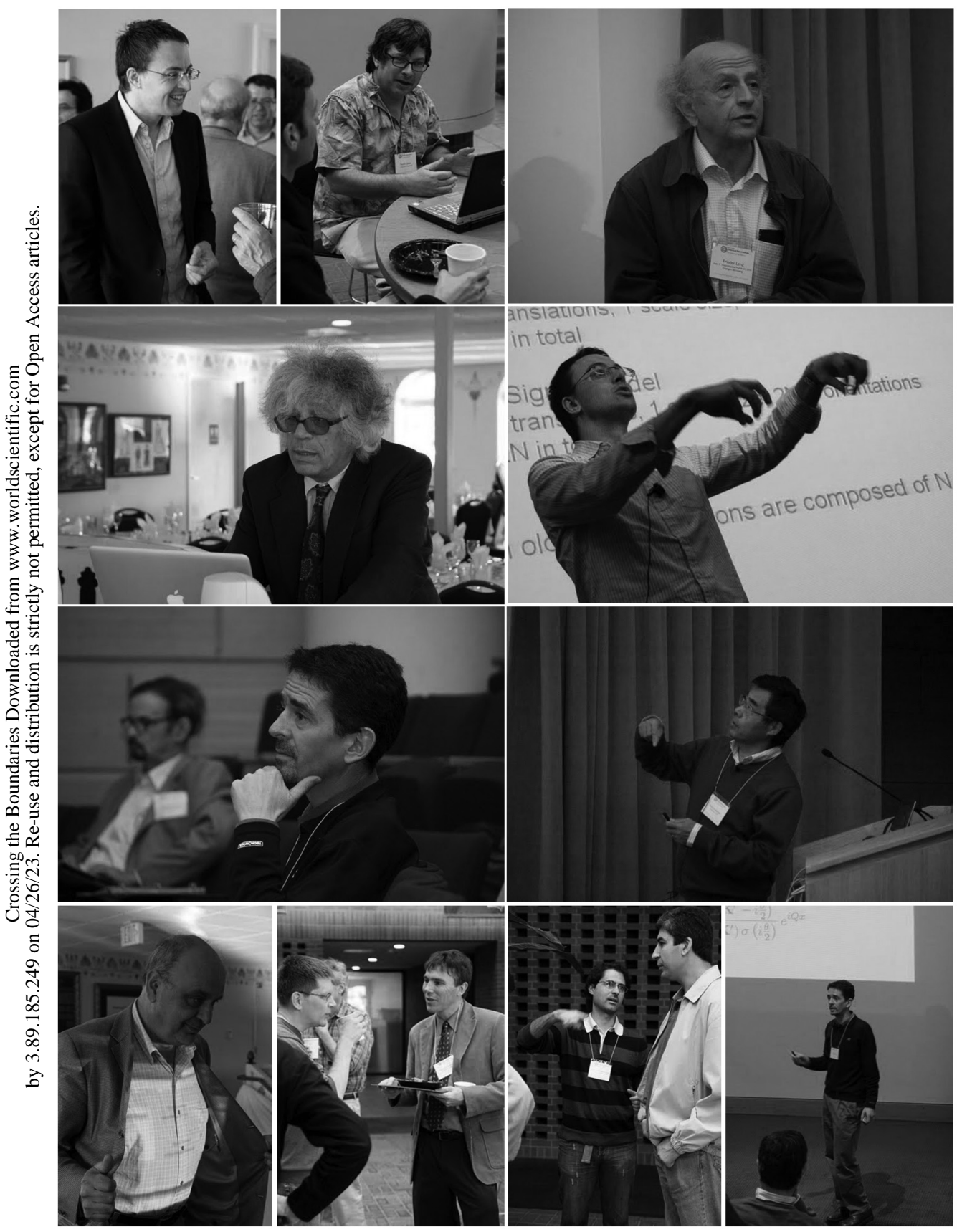




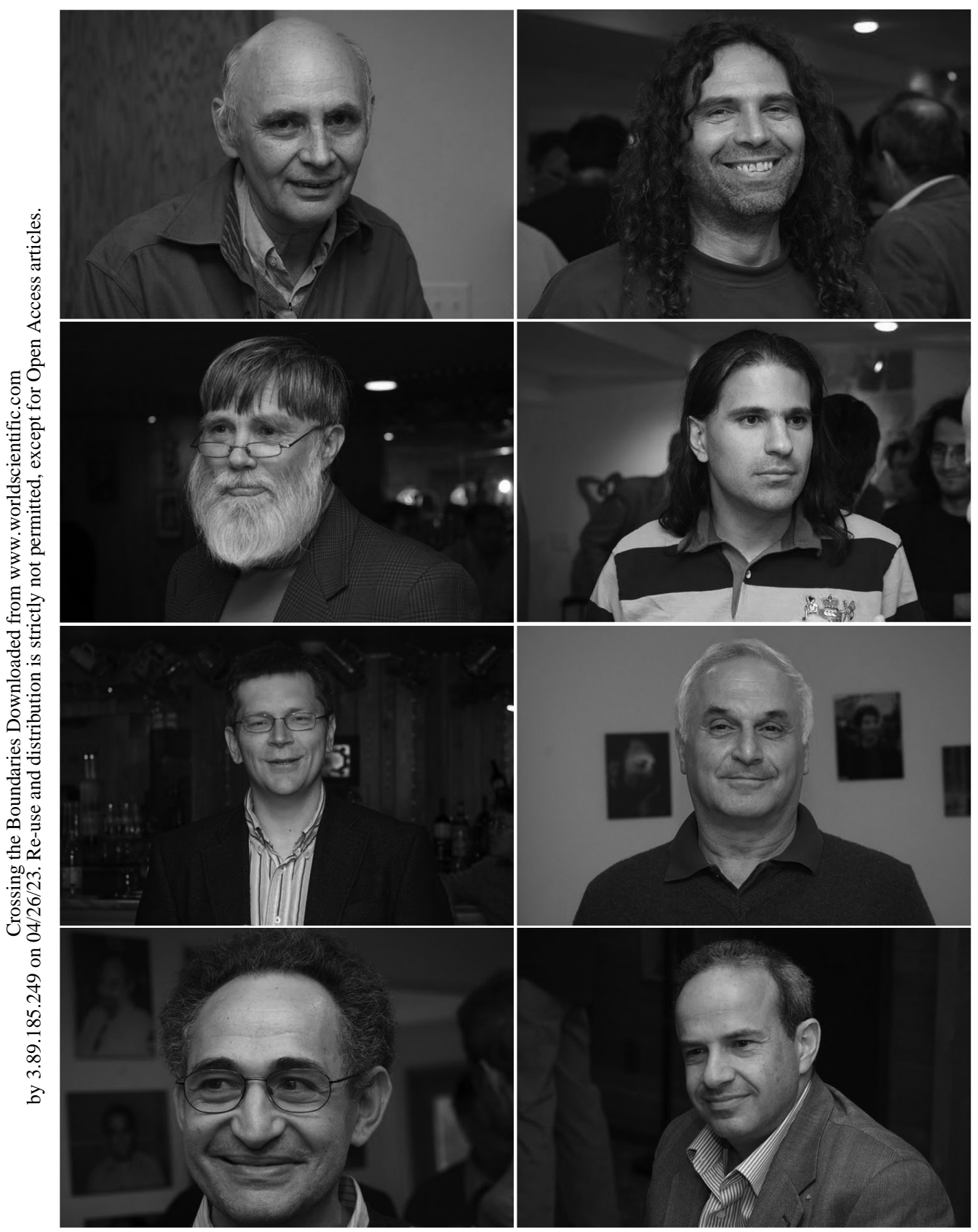




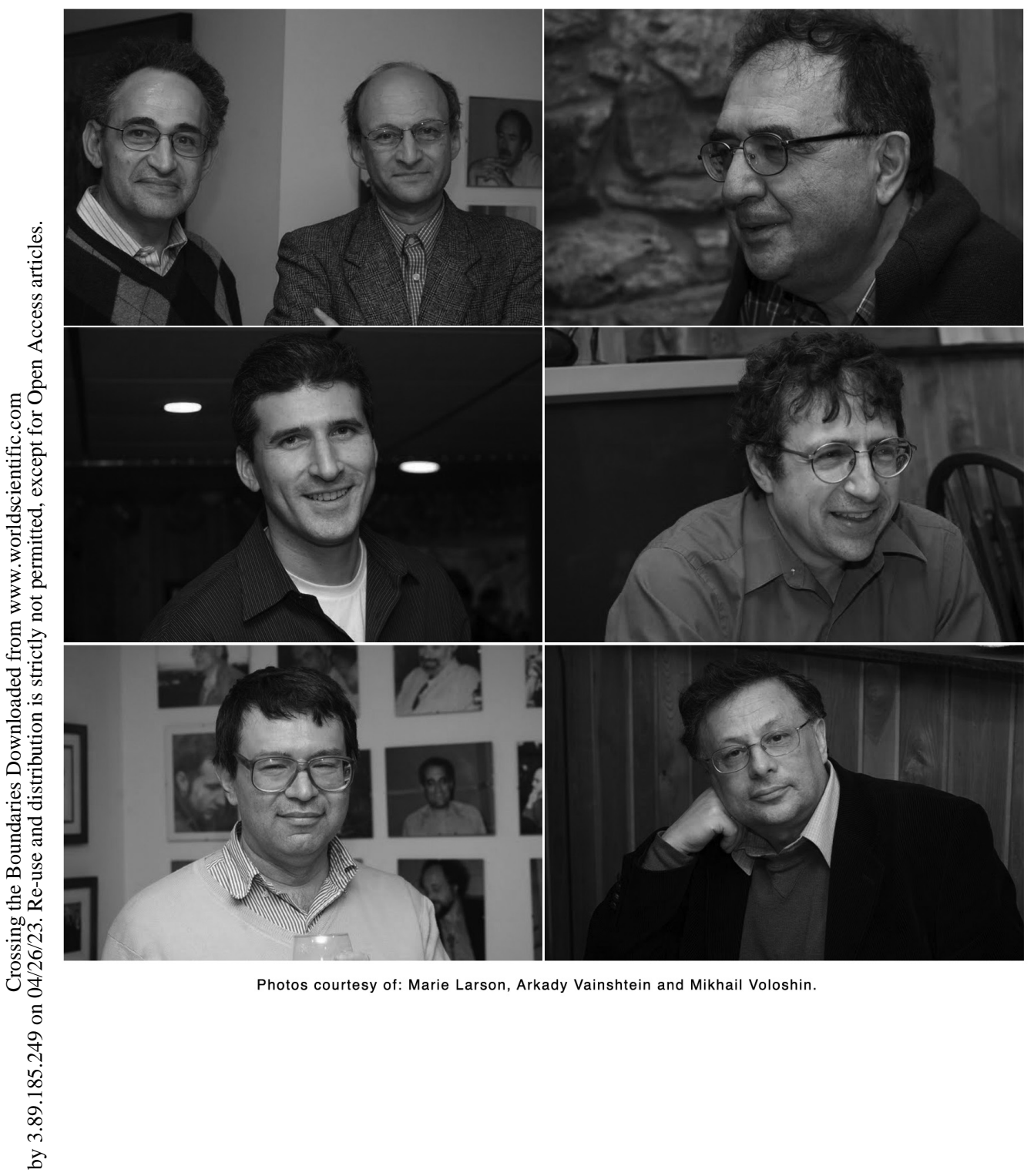


This page intentionally left blank 


\section{LIST OF THE PARTICIPANTS}

Nima Arkani-Hamed, Institute for Advanced Study

Roberto Auzzi, Swansea University

Ian Balitsky, Old Dominion University, Jefferson Lab

Viatcheslav Belyaev, Institute for Theoretical and Experimental Physics

Zvi Bern, University of California, Los Angeles

Tirthabir Biswas, Penn State University

Stefano Bolognesi, University of Minnesota, FTPI

Pavel Bolokhov, University of Pittsburgh

Thomas Cohen, University of Maryland

Xiaoyi Cui, University of Minnesota

Sergiy Dubynskiy, University of Minnesota

Gerald Dunne, University of Connecticut

Joshua Erlich, College of William and Mary

Harald Fritzsch, Ludwig-Maxmilians University, Munich

Gregory Gabadadze, New York University

Marcos Alejandro, Garcia Garcia Instituto de Ciencias Nucleares, UNAM

Stephen Gasiorowicz, University of Minnesota, FTPI

Howard Georgi, Harvard University

Ivan Gordeli, University of Minnesota

Elena Gubankova, Tufts University

Emir Gumrukcuoglu, University of Minnesota

Amihay Hanany, Imperial College, London

Burak Himmetoglu, University of Minnesota

Ken Intriligator, University of California, San Diego

Joe Kapusta, University of Minnesota

Alexander Khodjamirian, University of Siegen

Igor Klebanov, Princeton University

Kenichi Konishi, University of Pisa

Gregory Korchemsky, Institut de Physique Théorique

Peter Koroteev, University of Minnesota

Alex Kovner, University of Connecticut 
Lev Koyrakh, St. Jude Medical

Frieder Lenz, Inst. f. Theoretische Physik III, Univ. Erlangen-Nürnberg

Juan Carlos Lopez Vieyra, Instituto de Ciencias Nucleares, UNAM

Alexander Monin, University of Minnesota

Keith Olive, University of Minnesota, FTPI

Serkay Olmez, University of Minnesota

Sener Ozonder, University of Minnesota

Marco Peloso, University of Minnesota

David Politzer, California Institute of Technology

Maxim Pospelov, University of Victoria

Jose A. R. Cembranos, University of Minnesota, FTPI

Anatoly Radyushkin, Old Dominion University, Jefferson Lab

Pierre Ramond, University of Florida

Adam Ritz, University of Victoria

Serge Rudaz, University of Minnesota

Gregory Ruffa, University of Minnesota

Chris Savage, University of Minnesota, FTPI

John Schwarz, California Instutute of Technology

Nathan Seiberg, Institute for Advanced Study

Mikhail Shifman, University of Minnesota, FTPI

Edward Shuryak, Stony Brook University

Pierre Sikivie, University of Florida

Dam Son, University of Washington

Mikhail Stephanov, University of Illinois, Chicago

Raman Sundrum, The Johns Hopkins University

Tonnis ter Veldhuis, Macalester College

David Tong, University of Cambridge

Arkady Tseytlin, Imperial College, London

Alexander Turbiner, Instituto de Ciencias Nucleares, UNAM

Mithat Unsal Stanford University, SLAC

Alex Ushveridze, Capella University

Arkady Vainshtein, University of Minnesota, FTPI

Misha Voloshin, University of Minnesota, FTPI

Mark Wise, California Institute of Technology

Ariel Zhitnitsky, University of British Columbia 